\title{
Study on Unconstrained Compound Optimization Problem and Some Dual Problems of DC Optimization Problem with Compound Function
}

\author{
Liu Zhengrong \\ College of Science, Guangdong Preschool Normal College in Maoming, Maoming, Guangdong, 525000, \\ China \\ email: liuzhengrong555@163.com
}

Keywords: Composite Approach Beam Method, Linear Approximation, Dual Space, Composite Function

\begin{abstract}
There are many different solutions to non smooth optimization problems. In fact, the solutions of non smooth optimization problems can be divided into two main types: sub gradient method and beam method. Any method is based on the assumption that the objective function value is unique and there is a lower gradient value at each point. The sub gradient method is a method to solve the non smooth problem based on the sub gradient. This method is simple and easy to install, but may cause convergence, optimal detection and sub gradient approximation. The connection method is the ideological basis of beam method. Based on the information obtained from the black box, a piecewise affine model of the objective function in the original problem is constructed. According to the tangent plane method, the general beam method is generated. This method is now widely used. This can keep relevant information, such as repeat points, generate sub gradients through previous repetition, and create information packages, namely bundles. Therefore, the previously established information can be used in the current iteration step to generate the next iteration point. However, with the increase of the number of repetitions, the information beam expands more and more, which leads to huge computing and storage problems. In order to solve this problem, a special integrated compression technology has appeared in beamforming.
\end{abstract}

\section{Introduction}

As we know, many problems in the real world are to solve a specific optimization problem model, that is to find the maximum or minimum value of a function under a specific assumption. The models of these optimization problems are very simple, but it is very difficult to solve them without additional conditions. Optimization is not only a very practical topic, but also one of the core issues of modern scientific computing. At present, many kinds of optimization models are studied in depth, most of them are convex optimization models[1]. Therefore, the research of convex optimization has attracted many scholars' attention and research at home and abroad. Especially from 20 to 30 years, convex optimization has been studied the most widely, the theoretical research is in-depth, the application is more and more extensive, and the research team is growing. In addition, the research of compound convex optimization (that is, the compound whose objective function is convex function) has also been widely concerned and evaluated by many scholars. The basic reason is that, optimization, convex optimization, objective function of convex function and linear operator and many complicated optimization problems such as constrained optimization, adopt the special situation and idea of convex optimization, but on the other hand, many practical optimization location problem models, traffic problems, economic benefits and other applications, including comprehensive convex function. At present, many researchers have carried out in-depth research on the compound convex optimization problem and achieved many important results.

\section{Overview of Scalar Optimization and Vector Optimization}

However, all practical problems are accompanied by convex optimization or compound convex 
optimization[2]. One of the best examples of describing this situation is the problem of geographical location. For example, when buying a house, we should first consider the location of the house. On the other hand, the residence must be close to the supermarket, bus station and other specific departments. On the other hand, a house is a DC function (that is, the difference between two convex functions), which should be far away from specific departments such as chemical factories, airports, railway stations, etc. This problem is called DC optimization problem. After these practical problems are transformed into optimization models, the objective function and constraint function include DC function. Another classical model of nonconvex optimization is fractional optimization. At present, many optimization problems are the practical application problems of investment return and dividend coverage, production plan, logistics support, data mining, entropy optimization, and many optimization problems of fractional form transformation, so it is necessary to study.

$$
\begin{aligned}
& K=\left\{y \square Y:\left[y_{1}, y\right] \geq 0, \square y \square K \square Y\right\} \\
& y_{1} \leq_{k} y_{2} \square y_{2} \quad y_{1} \square K \\
& \partial_{\varepsilon} l(x)=\left\{x \square X: l(x) \geq l x+\left[\begin{array}{ll}
x, x & x
\end{array}\right] \quad \varepsilon\right. \\
& \partial l(x)=\left\{x \square X: l(x) \geq l(x)+\left[\begin{array}{ll}
x, x & x
\end{array}\right]\right. \\
& l(x)+l(x) \leq[x, x]+\varepsilon \\
& \text { epil }=\left\{x,[x, x]+\varepsilon \quad l(x): \quad x \varepsilon \partial_{E} l(x)\right.
\end{aligned}
$$

Figure 1 Consensus of composite function

\section{The Above Descriptions are All About Scalar Optimization}

However, in all kinds of decisions, such as economic operation, production, life, development and sustainable development of society, national defense, national security and so on, there are often more than two evaluation indexes, such as production process, economic growth, maximum benefit and minimum cost of environmental protection[3]. For these problems, multiple evaluation indexes need to be considered at the same time when making decisions. That is to say, the index system used to evaluate "good" or "bad" is not single but multiple. However, the indicators of this kind of problem are contradictory, so the adjustment of these contradictions, taking into account the indicators, finally choose the most ideal solution is that people often need to solve problems in real life, vector optimization problem should be applied[4]. Vector optimization is an important branch of new optimization theory and application. Its research field is mainlyparameter vector optimization problem, (parameter) set-valued optimization problem, (parameter) vector variational inequality problem, (parameter) vector balance problem, including vector inequality and other problems. Scholars at home and abroad from the mathematical model, network economy, decisionmaking, game theory and many other mathematical models in the real world, vector optimization problem is widespread Many problems in the analysis of the linear form are closely related. Setvalued analysis and variational analysis greatly expand the scope of theoretical research and application of vector optimization problems. For the development process and current situation of vector optimization, please refer to the literature and charts. In 1994, Blum and oettli studied the theory of two pairs of equilibrium problems and pointed out the mathematical model called equilibrium problems. The equilibrium problem provides a unified framework of mathematical planning problem, variational inequality problem, partition problem, fixed point problem, Nash equilibrium and complementary problem. Since then, many researchers have carried out in-depth research on this issue, and achieved rich research results. At present, the theoretical research on the equilibrium problem mainly includes the expansion of the model, the existence of solutions, and the relationship between the equilibrium problem and other problems.

\section{In this Paper, We Study the Generalized Differentiability of the Correlation Graph and the Double Pair Theory of the Optimization Problem}

Here is a brief summary of the story[5]. The dual problems of general equilibrium problems 
based on DC function are established, and the corresponding weak and strong pairs are obtained. The Farkas type results of general equilibrium problems are studied by using the double pair theory. Finally, the solution of generalized equilibrium problems is applied to convex optimization problems and generalized variational problems.

In locally convex space, the fractional optimization problem is transformed into a constrained optimization problem by dinkelbach method. Then, the graph property of conjugate function is used to introduce the closed condition, and its corresponding properties are discussed[6]. Finally, the Farkas type results of Faraday optimization are studied by studying the dual problems restricting the optimization.

In this paper, the dual problem of the compound convex optimization problem is introduced in the locally convex space[7]. Secondly, on the premise that the objective function of the complex convex optimization problem is not semi continuous and the constraint set is not necessarily closed, some constraint properties are introduced and discussed by using the properties of the curve graph of the conjugate function[8]. Finally, according to the strong bipartite, stable strong bipartite and all bipartite constraints of the complex convex optimization problem, we characterize them.

First, the studniarski derivative of set-valued mapping is imported to study the higher-order optimality conditions. Then, we study the relationship between the studniarski derivative functions of set-valued graphs and their profile graphs. Finally, the sensitivity of the derivative function of the perturbation map is obtained.

The calculation method of the quadratic dependent function of the sum of two set-valued graphs and the combination of two sets of mappings are studied. Then, the quadratic sensitivity of parameter set-valued optimization problem is studied by using the calculation method, and the quadratic dependent function of disturbance mapping and the specific formula of weak disturbance mapping of parameter set-valued optimization problem are obtained. Finally, we study the optimal solution mapping and generalized secondary subordinate superior derivative function of the optimal solution mapping. The corresponding result contains the previous result, which is generalized. The duality theory of generalized equilibrium problem, some new results are obtained, such as the calculation and generation problem, the compound convex optimization problem, the Farkas type of optimization, the higher-order optimality condition of the result set-valued optimization problem and the mutual dependence of the higher-order optimal condition parameter vector optimization problem, the sensitivity problem of the quadratic derivative of the blueprint and the upper derivative of the general quadratic subordination problem, and some new results are also given Step research has many shortcomings and problems.

\section{Conclusion}

The Farkas type results of fractional optimization problems can be obtained under the assumption of semi continuity and closure conditions[9]. However, in many practical problems, the discontinuity of correlation functions or the failure of closure of correlation sets are often encountered. Therefore, it is necessary to further consider Farkas type results of phase optimization without assuming topology.

Although we get the double problems of compound convex optimization, it is necessary to continue to study the double problems of multiple optimization models. For example, the method of studying this problem can be applied to the two pair problem of DC infinite plan with constraints. Assuming that there is no topological structure, we use the new pairing to study the strong pairing, stable strong pairing and complete pairing of DC infinite problems. In addition, inverse pairing can also be studied.

Although the expression of the generalized quadratic dependent graph derivative function of the best solution mapping and the best value mapping of the parameter vector optimization problem is investigated, the application of the generalized quadratic dependent graph derivative function is not discussed. In addition, it is necessary to consider the relationship between the derivative functions of generalized quadratic dependent curves and the derivative functions of various newly introduced generalized quadratic dependent curves. 


\section{Acknowledgements}

This research has been financed by The natural science Research Project in 2019 of the Guangdong Preschool Normal College In Maoming " Unconstrained optimization problem and its related research" (2019GMYSKT91).

\section{References}

[1] Kensuke Sekihara, Yoshiaki Adachi, Hiroshi K Kubota,. Beamspace dual signal space projection (bDSSP): A method for selective detection of deep sources in MEG measurements. Journal of Neural Engineering, vol. 15, no. 3, pp. 036026, 2018.

[2] Siamak Mehrkanoon, Johan A. K. Suykens. Regularized Semipaired Kernel CCA for Domain Adaptation. IEEE Transactions on Neural Networks \& Learning Systems, no. 99, pp. 1-15, 2017.

[3] Filippo Gandino, Renato Ferrero, Maurizio Rebaudengo. A Key Distribution Scheme for Mobile Wireless Sensor Networks: \$q - \$s \$ -Composite. IEEE Transactions on Information Forensics \& Security, vol. 12, no. 1, pp. 34-47, 2017.

[4] Wang B, Feng J E, Min M. Matrix approach to model matching of composite asynchronous sequential machines, vol. 11, no. 13, pp. 2122-2130, 2017.

[5] Jianghua Guo, Dongyan Shi, Qingshan Wang,. A domain decomposition approach for static and dynamic analysis of composite laminated curved beam with general elastic restrains. Mechanics of Advanced Materials \& Structures, pp. 1-13, 2018.

[6] Debabrata Das. Free vibration and buckling analyses of geometrically non-linear and sheardeformable FGM beam fixed to the inside of a rotating rim. Composite Structures, no. 179, 2017.

[7] Taruya Atsushi, Saga Shohei, Breton Michel-Andrès,. Wide-angle redshift-space distortions at quasi-linear scales: cross-correlation functions from Zel'dovich approximation. Monthly Notices of the Royal Astronomical Society, 2019.

[8] Chenchen $\mathrm{Wu}$, Donglei Du, Dachuan Xu,. Primal-dual approximation algorithms for submodular cost set cover problems with linear/submodular penalties. Numerical Algebra Control \& Optimization, vol. 5, no. 2, pp. 91-100, 2017.

[9] Zakaria Ilyes Djamai, Myriam Bahrar, Ferdinando Salvatore,. Textile reinforced concrete multiscale mechanical modelling: Application to TRC sandwich panels. Finite Elements in Analysis \& Design, no. 135, pp. 22-35, 2017. 